\title{
Value of TCD Foaming Test in Screening Patent Foramen Ovale in Migraine Patients and Efficacy of PFO Occlusion
}

\author{
Lilan Dü, Xiaorong Guo", Honglin Zhang, Min Zhang, Guangli Zou* \\ Department of Neurology, Chongqing General Hospital, University of Chinese Academy of Sciences, Chongqing 401147, \\ China \\ ${ }^{\#}$ Lilan Du and Xiaorong Guo are the frist authors and contributed equally to this work.
}

Abstract: Objective: To explore the effect of TCD
foaming test in screening patent foramen ovale in
migraine patients, as well as in the treatment effect. Methods: From September 2019 to August 2020, 236 patients with migraine and 362 patients with normal physical examination were treated in our hospital. According to the random number table method, 60 patients with migraine were selected as the observation group, and 60 patients with normal physical examination were selected as the control group. 48 cases of PFO were confirmed by TCD foaming test Among the patients with migraine, 36 patients received interventional occlusion therapy. The therapeutic effect and VAS score of the two groups were analyzed. Results: Through TCD foaming test, the proportion of PFO in the observation group was $80 \%$, and that in the control group was $13.33 \%$. The proportion of PFO in the observation group was significantly higher than that in the control group, and the difference was statistically significant $\left(\chi^{2}=53.5714, P<0.01\right)$; The symptoms disappeared in 30 patients and improved in 4 patients; Among the 36 patients who received interventional occlusion therapy, the VAS score of patients before and after the operation was significantly changed, and the proportion of patients whose pain disappeared after the operation was $83.33 \%$, which was significantly lower than that before the operation, and the difference was comparable $\left(\chi^{2}=51.4286, P<0.01\right)$. Conclusion: TCD foaming test for PFO screening has strong applicability, not only safe and convenient, will not cause trauma to patients, and through interventional occlusion treatment, can significantly improve the clinical symptoms of migraine patients and cure patients.

Key words: Migraine; TCD foaming test; Patent foramen ovale; Curative effect

Publication date: March, 2021

Publication online: 31 March, 2021

*Corresponding author: Guangli Zou, woshiyi zhixiaoql@sina.com

Migraine is a common head disease, characterized by severe headache, pulsation on one or both sides, often accompanied by nausea, vomiting, photophobia and noise on one side of the head. It is defined as chronic neurovascular disease. The disease usually begins in adolescence and reaches its peak in young and middle age ${ }^{[1]}$. There are frequent seizures and severe symptoms. There are more women than men, and among other inherited diseases, depression, anxiety, epilepsy, asthma and migraine are more common. A large number of studies have shown that there is a close relationship between migraine and PFO. The PFO of migraine patients is more than twice that of healthy people ${ }^{[2]}$. TCD foaming test is the most effective and accurate screening method for PFO, while percutaneous puncture and occlusion are the effective treatment methods for migraine in PFO. This paper studies the diagnostic value of TCD foaming test in screening PFO in migraine patients and the clinical effect of percutaneous puncture and occlusion in the treatment of migraine. 


\section{Data and methods}

\subsection{General information}

From September 2019 to August 2020, 236 patients with migraine and 362 patients with normal physical examination were treated in our hospital. According to the random number table method, 60 patients with migraine were selected as the observation group, and 60 patients with normal physical examination were selected as the control group. There were 28 males and 32 females in the control group; The average age was $(48.91 \pm 3.56)$ years (range, 3660 years). There were 25 males and 35 females in the observation group; The average age was (47.62 \pm 3.66 ) years (range, 35-59 years). There was no significant difference in the general information between the two groups $(\mathrm{P}>0.05)$. This study has been approved by the ethics committee, and patients have been informed and supported before the study. Informed consent has been signed by patients. All patients in the study are over 20 years old, 70 years old and below, and their basic symptoms meet the criteria of migraine. Patients in pregnancy and lactation, patients with infectious diseases, and patients with mental illness are excluded Patients with transsexual diseases, patients with severe mental disorders and cardiovascular diseases, and patients with contraindications should be excluded.

\subsection{Methods}

(1) TCD foaming test. The subjects in the two groups were supine on the bed, and a cuboidal vein channel was established, which was connected to a threeway tube. One end was connected to a syringe of 8 $\mathrm{ml}$ normal saline $+1 \mathrm{ml}$ air, and the other end was connected to a syringe of $1 \mathrm{ml}$ blood. The middle cerebral artery (MCA) was monitored by single channel and double depth single body with the depth of $48-60 \mathrm{~mm}$ [3]. Connect two $10 \mathrm{ml}$ syringes ( 8 $\mathrm{ml}$ normal saline, $1 \mathrm{ml}$ air, $1 \mathrm{ml}$ blood) on the tee tube, infuse once at rest, 2-4 times under tile action, then mix the mixed saline and air fully, and then inject the activated normal saline into the blood vessel (2-3 seconds). For negative patients, Valsalva maneuver was performed by reinjection of the bolus into the mixed air-water mixture for 5 seconds to closely monitor the microbubble signal. The positive results were based on the criteria recommended by the Venice conference in 1999, and the appearance of microbubble signal (MB) within 25 seconds was used as the diagnostic criteria. The number of microbubbles is type INo MB (voice result), class II:1-10 MB, class III:11-25mb, but no rain curtain. IV: Form a rain curtain. The positive patients in the observation group were diagnosed as tee and head $\mathrm{CT}^{[4]}$.

(2) 3 months after operation, TCD patients underwent TCD foam test and TTE test [4] combined with transthoracic echocardiography (TTE). The TCD method is the same as above. Meanwhile, the TTE probe is placed on the apex to obtain the four chamber heart image, complete the TCD foam test, and observe whether there is microbubble signal on the left side of the ventricle, the system and the middle cerebral artery.

(3) PFO blocking method. In the observation group, the patients who were diagnosed as PFO and willing to accept occlusion treatment were treated by percutaneous puncture and occlusion ${ }^{[5]}$. The delivery candle and PFO occluder produced by Beijing Huayi Shengjie company were selected. After femoral vein puncture, the $6 \mathrm{~F}$ right cardiac catheter and guide wire were sent to the right atrium, and the guide wire and guide wire were inserted into the left atrium from the orifice. After the hard guide wire was replaced, the ovary and left superior pulmonary vein were transferred from inferior vena cava, right atrium and bulbar ovary to left atrium. The PFO occluder was sent for occlusal treatment, and the occluder was confirmed by chest ultrasound. If it is good and stable, and does not affect the function of the heart valve, release the occlude, and take aspirin and clopidogrel orally after operation, aspirin $100 \mathrm{mg} / \mathrm{D}$, clopidogrel $75 \mathrm{mg} / \mathrm{D}, 6$ times a day. TCD foam test was performed on occlusal patients 1 months and 3 months after operation, and TTE and head CT scan were performed ${ }^{[6-8]}$.

\subsection{Observation indexes}

The results of TCD foaming test were compared between the two groups. The treatment effect and VAS score of the two groups before and after operation.

\subsection{Statistical methods}

Measurement data were expressed as mean \pm standard deviation $(\bar{x} \pm s)$, and $\mathrm{t}$ test was used. Enumeration data were expressed as rate (\%) and $\chi^{2}$ test was used. $P<0.05$ indicates that the difference is statistically significant. 


\section{Results}

\subsection{Comparison of general information}

In the general data, the proportion of male patients in the control group was $46.67 \%$, and that of female patients was $53.33 \%$. In the observation group, the proportion of male patients was $41.67 \%$, and that of female patients was $58.33 \%, P=0.5813$. The age of the control group and the observation group was tested, $t=1.9570, P=0.00527$. The results showed that the general data of the two groups were not statistically significant, and the experimental results were comparable, as shown in Table 1

Table 1. Comparison of half data between the two groups $[\bar{x} \pm s, n(\%)]$

\begin{tabular}{ccccc}
\hline \multirow{2}{*}{ Group } & \multirow{2}{*}{ Number of cases } & \multirow{2}{*}{ Age } & male & Gender \\
\cline { 3 - 5 } & & & $28(46.67)$ & $32(53.33)$ \\
control group & 60 & $48.91 \pm 3.56$ & $25(41.67)$ & $35(58.33)$ \\
$t$ & 60 & 1.9570 & & \\
$p$ & & 0.00527 & 0.5813 & \\
\hline
\end{tabular}

2.2 Comparison of TCD foaming test results between two groups

Through TCD foaming test, the proportion of PFO in the observation group was $80 \%$, and that in the control group was $13.33 \%$. The proportion of PFO in the observation group was significantly higher than that in the control group, and the difference was statistically significant $\left(\chi^{2}=53.5714, P<0.01\right)$. The experimental results were comparable, as shown in Table 2.

Table 2. The results of TCD were compared between the two groups

\begin{tabular}{cccccc}
\hline Group & Grade I & Grade II & Grade III & Grade IV & PFO \\
\hline Observation group $(\mathrm{n}=100)$ & $12(20.00)$ & $11(18.33)$ & $12(20.00)$ & $25(41.67)$ & $48(80.00)$ \\
Control group $(\mathrm{n}=100)$ & $52(86.67)$ & $3(5.00)$ & $3(5.00)$ & $2(3.33)$ & $8(13.33)$ \\
$\chi^{2}$ & & & & 53.5714 \\
$p$ & & & & 0.01 \\
\hline
\end{tabular}

2.3 Comparison of treatment effect between the two groups

In the observation group, 48 patients with confirmed PFO complicated with migraine were found, of which 36 patients received transcatheter closure therapy. The symptoms of 30 patients disappeared after operation, and the symptoms of 4 patients improved significantly. The patients needed to be followed up for 3 months after operation, and 2 patients received TCD again Foaming test, the experimental results are still left and right shunt, all patients were reexamined, no infarction was found in patients ${ }^{[9,10]}$.

\subsection{Compare the VAS scores of the two groups before and after operation}

Among the 36 patients who received interventional occlusion therapy, the VAS score of patients before and after the operation was significantly changed, and the proportion of patients whose pain disappeared after the operation was $83.33 \%$, which was significantly lower than that before the operation, and the difference was comparable $\left(\chi^{2}=51.4286, P<0.01\right)$, as shown in Table 3.

Table 3. Comparison of VAS scores before and after operation in 36 patients [ $n(\%)]$

\begin{tabular}{ccccc}
\hline Time & 0 points & $\mathbf{1 - 3}$ points & 4-6 points & $\mathbf{7 - 1 0}$ points \\
\hline Before operation & 0 & $18(50.00)$ & $12(33.33)$ & $6(16.67)$ \\
After operation & $30(83.33)$ & $4(11.11)$ & $2(5.56)$ & 0 \\
$\chi 2$ & 51.4286 & & & \\
$\mathrm{p}$ & $<0.01$ & & & \\
\hline
\end{tabular}

\section{Discussion}

Migraine is a common head disease, characterized by severe headache, pulsation on one or both sides, often accompanied by nausea, vomiting, photophobia and noise on one side of the head.It is defined as chronic neurovascular disease. The disease usually begins in adolescence and reaches its peak in young and middle 
age.Among them, Yu Xinyan ${ }^{[1]}$ and other studies show that TCD foaming test has a strong screening effect, which can effectively screen different types of patients and facilitate the treatment of patients; Liang Shimin ${ }^{[2]}$ pointed out in the study that patients with suspected PFO can be given priority to c-TCD or c-tte examination, and patients with positive examination results can be further given tee and c-tee examination $^{[11,12]}$.

The results of this study showed that among the 48 confirmed PFO patients with migraine, 36 patients received transcatheter closure therapy. The symptoms of 30 patients disappeared, and the symptoms of 4 patients improved significantly. The patients needed to be followed up for 3 months, and 2 patients received TCD again Foaming test, the experimental results are still left and right shunt, all patients were reexamined, no infarction lesions were found in patients, 36 cases of patients with interventional occlusion therapy, preoperative and postoperative VAS score significantly changed, postoperative pain disappeared accounted for $83.33 \%$, significantly lower than preoperative.

To sum up, TCD foaming test has strong applicability for PFO screening, which is not only safe and convenient, but also can not cause trauma to patients. Moreover, interventional occlusion can significantly improve the clinical symptoms of migraine patients and cure them.

\section{References}

[1] Wang WX, Wu HQ. Application of TCD foaming test combined with CTA in screening the etiology of ischemic stroke in young people [J]. Journal of Cardio Cerebrovascular Diseases of Integrated Traditional Chinese and Western Medicine, 2019,17 (04): 597-599

[2] Wang Sp, Huang WX, Wang D, Zheng HRi. Diagnostic value of TCD foaming test in migraine with patent foramen ovale [J]. Nerve Injury and Functional Reconstruction, 2018,13 (12): $652-653+661$
[3] The significance of TCD foaming test in screening young cryptogenic stroke patients with patent foramen ovale [E]. Cangzhou People's Hospital, Hebei Province, December 17, 2018

[4] Liu LL, Li X, Li SS, Wu G, Wu JH. Significance of TCD foaming test in etiological detection of unexplained young stroke patients [J]. Journal of Cardio Cerebrovascular Diseases of Integrated Traditional Chinese and Western Medicine, 2018,16 (18): 2729-2730

[5] Zhang CL, Gao HH, Li KX, Wang YX, Chen Y. Clinical significance of TCD foaming test in etiological diagnosis of cryptogenic stroke in young people [J]. Journal of Cardio Cerebrovascular Disease of Integrated Traditional Chinese and Western Medicine, 2018,16 (18): 2731-2732

[6] Zheng T. Nursing experience of transcranial Doppler foaming test in detecting patent foramen ovale in young stroke patients [J]. Medical Equipment, 2018,31 (18): 185-186

[7] Li D. Nursing analysis of TCD foaming test in the diagnosis of young stroke patients with patent foramen ovale [J]. International Medical and Health Guide, 2018,24 (17): 26952697

[8] Zhang Z. Observation on the short-term effect of transcatheter closure of patent foramen ovale in 33 cases [D]. Shandong University, 2018

[9] Wang JJ. The predictive value of vascular ultrasound on the efficacy of intravenous thrombolysis in patients with ischemic stroke [D]. Xinxiang Medical College, 2018

[10] Yuan K. Correlation between patent foramen ovale and ischemic stroke [D]. Nanhua University, 2018

[11] Chen WX, Huang MS, Chen HJ, Wu XL, Zhang Z2, Li X, Fang HM, Comparison of diagnostic value of transcranial Doppler ultrasound foaming test and transthoracic echocardiography in young and middle-aged cryptogenic stroke with patent foramen ovale [J]. Chinese Journal of Clinical New Medicine, 2018,11 (03): 234-237

[12] Xiao YL, Chen LF, Qin J, Huang QM, Wu CY, Li HM, Xiao K, Qin Y, LV HQ, Meng Y, Xie J. Safety analysis of TCD foaming test in clinic and possible clinical emergency measures [J]. Imaging Research and Medical Application, 2018,2 (08): 181-182 